\title{
Concentrate and Solvent for Concentrate for Oral Spray, Suspension Dosage Form
}

National Cancer Institute

\section{Source}

National Cancer Institute. Concentrate and Solvent for Concentrate for Oral Spray,

Suspension Dosage Form. NCI Thesaurus. Code C149375.

A concentrate and a solvent intended for the preparation of a concentrate for oral spray, suspension by diluting the concentrate with the solvent; the resulting concentrate for oral spray, suspension must subsequently be diluted again before administration as an oral spray, suspension. 